\title{
Trinken im öffentlichen Raum
}

\section{Eine kooperative Konfliktlösung ist möglich}

\author{
Ute Kinn
}

In many urban agglomerations, conflicts between civil interests and needs of disadvantaged people are often sparked off on public places. Although these conflicts may not be resolved by district management this approach was at least suitable to ease them in favour of the communities.

Dans beaucoup d'agglomérations urbaines, les conflits entre intérêts privés et personnes en situation d'exclusion se heurtent souvent violemment sur la place publique. Bien que ces conflits ne pourraient pas être résolus au niveau du quartier, ce niveau d'intervention s'est révélé assez pertinent pour les apaiser en faveur des habitants $d u$ quartier.

Ute Kinn von »GRiPS Büro für

Projektsteuerung und

Kommunikation « führt seit 2003 das Stadtteilmanagement für die Soziale Stadt Stuttgart Rot durch.

E-Mail ute.kinn@grips-ettlingen.de
In vielen städtischen Ballungszentren entzünden sich an öffentlichen Plätzen Konflikte zwischen bürgerlichen Interessen und Bedürfnissen von Menschen am Rand der Gesellschaft. Ein Konflikt, der durch kluges Stadtteilmanagement wenn nicht gelöst, so doch im Sinne des Gemeinwesens entspannt werden kann.

Im Stuttgarter Stadtteil Rot hat sich bislang keine harte Szene (Drogen, Alkohol, Gewalt, Kriminalität) mit unauflösbarem Gruppencharakter verortet, vielmehr treffen sich an verschiedenen öffentlichen Plätzen lose Gruppen, die mit zunehmendem Alkoholkonsum auffällig werden. Begleiterscheinungen wie Alkoholmissbrauch, Lärm und Verunreinigung stoßen beim Umfeld auf Distanz und Ablehnung. $\mathrm{Zu}$ Recht sorgen sich die Bewohner um das Image Ihres Stadtteils. Die dringlichste Aufgabe ist deshalb eine Beruhigung des schwelenden Konflikts zwischen auffälligen und nicht auffälligen Nutzern des öffentlichen Raums.

Der Stuttgarter Stadtteil Rot ist seit 2003 im Bund-Länder-Programm »Stadtund Ortsteile mit besonderem Entwikklungsbedarf - Die Soziale Stadt« (vgl. den Beitrag von Martin Holch in diesem Heft). Seit diesem Zeitpunkt erfolgt in den Arbeitsfeldern: »Kinder und Jugendliche «, »öffentliche Plätze und Verkehr «, "soziales und kulturelles Miteinander" sowie »Wohnen und Wohnumfeld « in Rot zügig die Planung und Umsetzung von Projekten aus der offenen Bürgerbeteiligung.

Die Projektgruppe »Trinken im öffentlichen Raum « der Sozialen Stadt Rot befasst sich seit Mai 2004 mit der Thematik. Ihr gehören Vertreterinnen und Vertreter der Bürgerschaft, der Evangelischen Gesellschaft Stuttgart, der städtischen Ämter, der Neuen Arbeit, der Evangelischen Kirchengemeinde Rot, der Wohnbaugesellschaften und des Polizeireviers Stuttgart-Zuffenhausen, ein Architekt und Künstler sowie das Stadtteilmanage- ment an. Die Projektgruppe wird im Rahmen der »Sozialen Stadt « vom Stadtteilmanagement für Rot moderiert. Die Projektgruppe hat folgende Ziele für eine Konfliktlösung formuliert:

- Die Situation der durch Alkohol auffälligen Männer und Frauen in Rot soll verbessert werden.

- Es sollen Methoden erprobt werden, die Ängste abbauen, Konflikte entspannen, einen Interessensausgleich schaffen.

- Aktionen werden initiiert, die das Image des Quartiers verbessern.

Neben der gemeinsamen Zielsetzung wurden von der Projektgruppe Maßnahmen in die Wege geleitet, die helfen sollen, den schon viele Jahre gärenden Konflikt zu entschärfen. Bei der Realisierung der kurzfristig angestrebten Maßnahmen konnte auf Fördermittel aus dem Programm »Lokales Kapital für Soziale Zwecke« (LOS) des Europäischen Sozialfonds zurückgegriffen werden.

Der Projektgruppe war es vor allem wichtig, eine solide Datenbasis für die Arbeit am Thema zu schaffen. Das Stadtteilmanagement der »Sozialen Stadt « hat deshalb, im Rahmen eines LOS-Projekts, eine Studie beauftragt und begleitet, um wesentliche Fragestellungen in direktem Kontakt mit der Zielgruppe zu beantworten:

- Wo und wie leben die Menschen, die sich in Rot an öffentlichen Plätzen durch starken Alkoholkonsum und sozial auffälliges Verhalten ins Bewusstsein rufen?

- Was lässt sich über Alter, Geschlecht, berufliche Ausbildung sagen?

- Welche Aufenthaltsorte in Rot werden verstärkt aufgesucht, wo handelt es sich eher um Randerscheinungen?

- Welche Möglichkeiten und Grenzen eines allseits akzeptierten Treffpunkts sehen die Betroffenen selbst?

- Wie groß ist die Bereitschaft, sich im Stadtteil nützlich zu machen, beispielsweise in einem Arbeitsprojekt oder in einer Tauschbörse? 
- Besteht ein Bedarf an niederschwelligen Beratungs- und Anlaufstellen vor Ort? Aus der Gruppe der »öffentlichen Trinker« konnten dreißig Männer ausführlich befragt werden. Die in der Studie dokumentierten Ergebnisse liegen seit Juni 2005 vor. Sie waren für die weiteren Schritte von großer Bedeutung. Durch die Befragung konnte sich die Projektgruppe ein klares Bild von den Beweggründen und Bedürfnissen der betroffenen Männer machen und die Projekte entsprechend ausrichten. Die suchtkranken Männer selbst haben bei der Befragung in ermutigenderweise Interesse und Mitwirkungsbereitschaft bezeugt. Auf Basis der möglich, festgefahrene Fronten und Zuweisungen im Stadtteil aufzulösen. - Die suchtkranken Männer konnten der bürgerlichen Bevölkerung beweisen, dass sie zu koordinierter und konstruktiver Projektarbeit in der Lage sind, was für die weiteren Maßnahmen von großer Bedeutung war.

\section{»Grünservice Rot «}

Der »Grünservice Rot « begleitet den gesamten Konfliktlösungsprozess und ist eine Ergänzung zu den künstlerischen und städtebaulichen Lösungsansätzen.

\section{»Dringlichste Aufgabe ist eine Berubigung des schwelenden Konflikts zwischen auffälligen und nicht auffälligen Nutzern des öffentlichen Raums"}

Studie wurden drei weitere Projekte in die Wege geleitet: "Stühle in Rot«, »Grünservice Rot « und »DenkWirkstatt .

\section{»Stühle in Rot «}

Dem Künstler Wolfgang Zaumseil ist es gelungen, eine feste Gruppe aus den Bewohnern des Wohnheims IGH für eine künstlerische Annäherung an das Thema »Trinken im öffentlichen Raum « zu begeistern. In regelmäßigen Arbeitstreffen zwischen Januar und Juni 2005 wurden fantasievolle Stühle erschaffen. Damit wurden das "Sitzen ", das "sich Aufhalten " und das "sich Niederlassen " im öffentlichen Raum auf ungewöhnliche Weise dargestellt. Mit diesem Projekt konnten mehrere Ziele erreicht werden:

- Männer aus der Gruppe der »öffentlichen Trinker « konnten nachhaltig zur Mitarbeit gewonnen werden.

- Mit der Präsentation der Kunstwerke am 30. Juni 2005 im Stadtteil und am 27. September 2005 im Bezirksbeirat war ein Anlass gegeben, um mit dem Gesamtprojekt auf die Öffentlichkeit zuzugehen.

- Durch die spielerische und sensible Bearbeitung des Konfliktthemas war es
Mit diesem Angebot bewegen sich die suchtkranken Männer sehr stark auf das » bürgerliche« Rot zu.

Unter der Leitung der »Neuen Arbeit «, einem Unternehmen der Evangelischen Gesellschaft Stuttgart, werden die Bedürfnisse der Bevölkerung nach einem ordentlichen und sauberen Rot aufgegriffen: Jeden Tag ziehen die Männer gegen ein geringes Handgeld los und reinigen im Auftrag des Garten-, Friedhofs- und Forstamtes öffentliche Flächen und Grünanlagen von besonderen Verschmutzungen. Dabei ist die Zusammensetzung der Truppe wechselhaft und unverbindlich, entsprechend den unterschiedlichen Problemen, die die Männer haben. Dennoch wird deutlich, dass die Männer durchaus das ihrige dazu beitragen möchten und können, um den Stadtteil zu pflegen und zu unterstützen.

Derzeit gibt es von allen Seiten große Bemühungen, das Projekt dauerhaft für die Männer in Rot zu sichern, also von den LOS-Fördermitteln unabhängig zu machen.

\section{»DenkWirkstatt «}

Das Projekt versteht sich als planerische Fortführung der künstlerischen An- sätze des Projekts »Stühle in Rot «. Zusammen mit Männern, die sich gern im öffentlichen Raum aufhalten, entwickelte der Künstler und Architekt Wolfgang Zaumseil in wöchentlichen Treffen konkrete Vorstellungen zum Standort und zur Ausstattung eines Aufenthaltsbereichs in Rot. Ein Modell wurde gebaut, funktionale und künstlerische Ausstattungselemente wurden mit den Männern in einer provisorischen Werkstatt entworfen.

Zwischen Januar und Juni 2006 wurden die Zwischenschritte (Aufgabenstellung, Ausstattung, Gestaltung und Standort) in vier Kolloquien vorgestellt und mit eingeladenen Vertretern der Anwohner, des Stadtteils und der Fachstellen rückgekoppelt.

\section{Resümee}

Es scheint, dass in Stuttgart-Rot die großen Bemühungen aller Beteiligten in die richtige Richtung führen. Nachdem der Standort für einen Bereich, an dem die Männer sich aufhalten können, festgelegt worden ist, wurde dieses Zwischenergebnis wieder einer größeren Öffentlichkeit präsentiert. Sowohl bei einer Veranstaltung vor Ort wie auch bei der Vorstellung im Bezirksbeirat erhielt der eingeschlagene Weg durchweg $\mathrm{Zu}$ stimmung. 